\title{
The Mathematical Model And Dynamic Analysis Of Nonlinear Biochemistry System
}

\author{
Xiao Xiaonan \\ Xiamen University Tan Kah Kee College, Zhangzhou Fujian China \\ xiaoxn@xujc.com
}

Keywords: Nonlinear, Biochemistry system, Mathematical Model, Dynamic Analysis.

\begin{abstract}
This article, with the example of different circulation system for a kind of nonlinear, discusses how to make a dynamic analysis of a nonlinear biochemistry system in line whit the character of the system and intercommunication and interaction of each part, thus to establish a realistic ideal mathematics model so as to make numerical calculus. The method in this article is of some reference value to establish models of relative nonlinear differential biochemistry system numerical calculus.
\end{abstract}

\section{Introduction}

The relationships between protein concentration in plasma and the rate of filtered glomerulus: Taking into consideration the single glomerulus capillary's state, this kind of capillary lies in the afferens arteriola. Its cavity pressure is far bigger than the total of Bowman's bladder fluidic static pressure and plasma albumen osmotic pressure. It lets moisture and small molecule solute through capillary entering Bowman's bladder. Thus, one of the effects of filtered glomerulus is that it makes plasma lose some liquid. With the flood flowing the effective filtered pressure gradually reduces. On the one hand, it is because the resistance of capillary to fluidic makes cavity pressure drop; On the other hand it is because the filtering of the fluidic raises the concentration of plasma protein within capillary. Thus it raises osmotic pressure.

The idealized model of glomerulus capillary

In order to make ration research, we establish the idealized model of the following glomerulus capillary.

We combine several million glomerulus capillaries into one epuicalent tube, and let its character agree with the reastic value of experiment animal(dogs) glomerulus filter.

\section{The numerical analysis of glomerulus capillary nonlinear differential system}

We suppose:

Maintaining the rate of filtering of each area under each unit pure filtered pressure;

Maintaining the original radius of capillary;

Plasma protein can't leak out from capillary;

The amount of reducation of pressure is the same after passing by the capillary of unit length; 
Let fluidic static pressure winthin Bowman's pocket along with capillary maintain.

In the folloeing we consider that a section of capillary which the distance is $\mathrm{d} x$ from the beginning side $x$ of the capillary. The area Aof that annular interception wall is: $A=2 \pi \mathrm{rd} x$

Pure filtered pressure $P_{\text {filt }}(x)$ is: $P_{\text {filt }}(x)=P_{\text {cap }}(x)-P_{\text {onc }}(x)-P_{\text {Bow }}$

According to (1), blood current because of filtered through annular wall so aking into Bouman's pocket. It causes the amount of reducation, which is:

$$
-\operatorname{dv}(x)=\mathrm{kAP}_{\text {filt }}(x)
$$

We obtain on the basis of above-mentioned three forms:

$-\mathrm{dv}(x) / \mathrm{d} x=2 \pi \mathrm{rk}\left[P_{\text {cap }}(x)-P_{\text {onc }}(x)-P_{\text {Bow }}\right]$

Accoding to (4), we have:

$P_{\text {cap }}(x)=P_{\text {cap }}(0)-x / L\left[P_{\text {cap }}(0)-P_{\text {cap }}(L)\right]$

Scatchard has ever stuied the relationship between proein albumen within plasma and osmotic pressure, within the scope of which we consider pretein is less than $0.12 \mathrm{~g} / \mathrm{ml}$. We can indicate his experimental result (referring to the plasma) by using the following equation:

$$
P_{\text {onc }}(c)=r c^{2}+\delta c
$$

Where $\gamma$ and $\delta$ are all the parameter of fitting curve. Now we suppose scaichard's observation is also correct to dogs. Based on the definition, we have the following: $C(x)=Q / V(x)$

Due to (3), here $\mathrm{Q}$ is one pavameter unrelated to $x$. We put (2.7)into (2.6), we have:

$$
P_{\text {onc }}(C(x))=P_{\text {onc }}(x)=r[Q / V(x)]^{2}+\delta[Q / V(x)]
$$

According to (2.4), (2.5) and (2.8), we have:

$$
\begin{aligned}
& -\frac{\mathrm{dv}(x)}{\mathrm{d} x}=2 \pi \mathrm{rk}\left\{P_{c a p}(0)-x / L\left[P_{c a p}(0)-P_{c a p}(L)\right]\right. \\
& \left.\left.-r[Q / V(x)]^{2}-\delta[Q / V(x))\right]-P_{B o w}\right\}
\end{aligned}
$$

Therefore in (2.9) we just have three variables $x, \mathrm{~V}(x)$ and $\mathrm{dv}(x) / \mathrm{d} x$. Moreover three are some consants, but most of them are estimated by physiological experiments. How to define related value $\mathrm{k}, \mathrm{r}, x$ and $\mathrm{L}$ in capillary ideal model? At first when we consider $x$ and $\mathrm{L}$, we can use one independent: $\mathrm{X}=x / \mathrm{L}$

Which just has definite instead of $x$. Here $\mathrm{X}$ is the percentage of total length .As $\mathrm{X}$ is the ratio of unclimension, we needn't choose any measure unit in the capillary model (in the certain sense, we can regard $\mathrm{L}$ as length unit, therefore when $x=\mathrm{L}, \mathrm{X}=1)$. Due to $(2.10)$, we have: $x=\mathrm{LX}$, put it into (2.9), then we obtain: $-\mathrm{dv}(\mathrm{LX}) / \mathrm{d} x=\pi \mathrm{rk}\left\{P_{c a p}(0)-\mathrm{X} P_{c a p}(0)-\right.$ $\left.\left.P_{\text {cap }}(L)-r[Q / V(l x)]^{2}-\delta[Q / V L(X))-P_{B o w}\right]\right\}$

After that, we again give a new definition constant $\mathrm{B}: \mathrm{B}=2 \pi \mathrm{rLk}$

We put (2.12) into (2.11), and we have:

$$
\begin{gathered}
-\mathrm{d} v(L X) / \mathrm{d} x=B\left\{\left[P_{c a p}(0)-P_{B O W}\right]-\left[P_{c a p}(0)-P_{c a p}(L)\right] X\right. \\
\left.-r Q^{2} / V^{2}(L X)-\delta Q / V(L X)\right\}
\end{gathered}
$$

We then put Table I's related parameter Value into (2.13), we have:

$$
-\mathrm{d} v(L X) / \mathrm{d} x=B\left\{70-20 X-2500 Q^{2} / V^{2}(L X)-200 Q / V(L X)\right\}
$$

Considering that circumstance when plasma with $2 \mathrm{ml} / \mathrm{s}$ enters capillary. Hence bound condtion is: $\mathrm{V}(\mathrm{o})=2 \mathrm{ml} / \mathrm{s}$ 
Because of $\mathrm{V}(\mathrm{o})=2, c=1 / 15 \mathrm{~g}$ protein $/ \mathrm{ml}, \mathrm{Q}=2(1 / 15)$, there is $0.133 \mathrm{~g}$ protein entering the capillary each second, so (2.14) wll be

$$
\mathrm{d} v(L X) / \mathrm{d} x=B\left\{20 X+44.44 / V^{2}(L X)+26.67 / V(L X)-70\right\}
$$

In the normal dogs, filtered percentage (that is the filtered rate of glomerulus dividing "effective plasma current" is about 0.3 . In order to estimate parameter $\mathrm{B}$, we require: $\mathrm{V}(\mathrm{L})=$ $\mathrm{V}(\mathrm{o})-0.3 \mathrm{~V}(\mathrm{o})=1.4$

Which is the seclnd bound coneitio. Due to the fact that $\mathrm{B}$ is not convinent for us to estimate directly. We first elect some probably value of B. According to (2.16) and $\mathrm{V}(\mathrm{o})=2 \mathrm{ml} / \mathrm{s}$ we can solve $\mathrm{V}(\mathrm{L})$. Again comparing with that has been solved $\mathrm{V}(\mathrm{L})$. and $1.4 \mathrm{ml} / \mathrm{s}$, we see how far it is, then change the value of B according to the condition, repeat the above process until at last error falls in the permittable scope.

We can obtain the above process through computer with the best method. Meanwhile when the plasma albumen concentration is different, the dropping curve of blood current in capillary because of liquid filtering out along the capillary can also be pictorialized.

\section{Conclusion}

Every biochemistry system is actually a nonlinear system. There exist all kinds of complex communication and interaction in it. On account of the complexity of biochemistry in order to establish one mathematics model of scientific objective biochemistry system, we not only want to observe the static state of the system. More important, we still observe motive state of the system and analysis and study of the intercommunication of each part in the system, the characteristic of the total system, and interaction between the system and outside environment. After establishing mathematics model, to be in the view of exact, we still must correct the error between theory and reality gradually until the mathematic model keeps propv correspondence with biochemistry system.

\section{References}

[1] Xiao Xiaonan. The optimal non-liner filtering and majorized algorithm of a king of nonstationary stochastic transmission system.Joural of Mthematical Study , 2010, 43(4): 342-351.

[2] Qiu J;Zhang L.F-interfere law genercetion and its feature recognition.Jourmal of Systems Engineering and Electronics,2009,20(4):777-783.

[3] He Weilian. Permanence for single species model with feedback control and finite continuous delays Journal of Fuzhou University (Natural science Edition,2009,37(4):468-470

[4] Chen Lixin, Lin Guochen,Lan Yongyi,et al.Measure theory of satistical convergence.Science in China A:Mathematics ,2008,51:2285-2303.

[5] Chen F.D,Li Z,Huang Y.J..Note on the permanence of a competitive system with infinite delay and feedback controls[J].Nonlinear Anal. Real World Appl .,2007,8(2):680-687. 
[6] Dogru O,Duman O.Statistical approximation of Meyer-Konig and Zeller operators based on q-integers.Publ Math Debrecen,2006,68(1-2): 199-214.

[7] Zhang T , Ge S S,Hang C C. Stable adaptive control for a class nonlinear systems using a modified lyapunov function. In: proceedings of the 14th IFAC World Congress. Beijing: 1999.K:373-378

[8] Yu L,Chen G D, Chu J .Optimal guaranteed cost control of linear uncertain systems: LMI approach. IFAC 1999,G-2e-21-1:541-546

[9] Tijms H C . Stochastic Models, An Algorithmic Approach. John Wiley \&Sons,1994. $156-248$

[10]Anderson T w. An Introduction to Multivariate Statistical Analysis. New York: John Wiley \&Sons, 1990. 232-296

[11]Lucin L C. Asymptotic Methods in Statisical Decision Theory. New York: Spring-Verlag, 1988.264-298

[12]Korezlioglu H, Mazziotto G, Szpirglas J. Filtering and control of Random Processes. Berlin: Spring-Verlag,1984.164-198 